\section{THE METHOD OF ENERGY- EFFICIENCY INVESTIGATIONS OF THE NEWLY PRODUCED MARINE FUELS THROUGH AN APPLICATION OF THE LABORATORY DIESEL ENGINE}

DOI 10.2478/ntpe-2018-0044

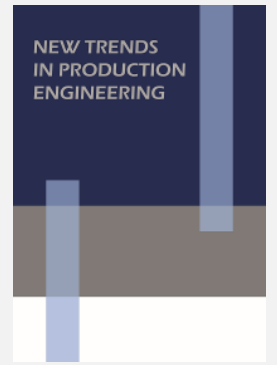

2018

Volume 1

Issue 1

pp. $357-363$

Gdansk University of Technology, Poland

\begin{abstract}
The article deals with one of the scientific issue solved within frames of the research project financed by Voivodeship Fund for Environmental Protection and Water Management in Gdansk, namely, in particular, the method of energy investigations of the self-ignition engine in conditions of its supply with a non-standard (modified) marine fuel. The representative results of engine tests worked out on the especially built laboratory test bed have been presented. They aimed to elaborate energy profiles of a single-cylinder four-stroke Diesel engine in the form of Sankey diagrams of energy flows representing the most efficient and effective manner for the verification of a different kind of the modified marine fuels introduced on market. It is expected that the final results of the project will be interesting for their producers as well as the ship-owners of the operated ships, and also for the offices of sea administration as well as the inspectorates of environment protection.
\end{abstract}

Keywords: modified marine fuels, engine research, Sankey's graphs

\title{
INTRODUCTION
}

The selected research results of the laboratory Farymann Diesel D10 engine fed with Ekodiesel ULTRA distillatory fuel (produced by PKN ORLEN S.A.) were introduced within this elaboration. The engine drives a direct current generator which powers a heating module of the "heavy" residual fuels - Fig. 1 (Korczewski et al., 2017a).

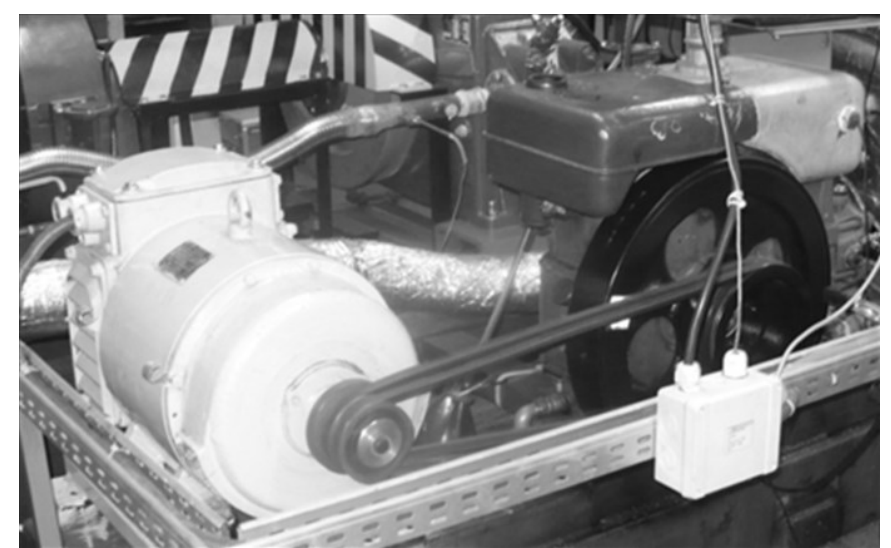

Fig. 1. General view of the laboratory test bed of a single-cylinder four-stroke Diesel engine that drives a direct current generator

The research is worked out within the frame of the wider investigative project funded by the Voivodeship Fund for Environmental Protection and Water Management in Gdansk. The research are aimed at the elaborating a calculation method of engine's energy profiles in conditions of feeding with non-standard (modified) marine fuels, during the engine's steady work on representative loads given according to propeller and regulatory profile (Korczewski et 
al., 2017b). In order to achieve the set research goal it was necessary to elaborate test programmers as well as measurement technology of the observed control parameters. Moreover, an adjusted set of the control parameters has to be minimalized to the extent enabling conduction of analogous investigations on real objects, i.e. marine engines, in their natural operation conditions. It was also established, that the engine's energy will be represented by a form of stream graphs, so called Sankey's graphs as well as developed indicator graphs and thermographs (Zeman and Castaldi, 2008; Abedin et al., 2013; Hürdoğan, 2016).

They enable an accomplishment of comparative analysis (qualitative) of the energy processes' course in an engine fed with different marine fuels engine, in appointed load's steady states (reference states).

The graphic visualizations of energy processes have been supplemented with detailed tabular listings that include numerical values of the measuring and computational parameters. They stand for a basis of the quantitative assessment of an impact of values of the applied fuel's physico-chemical parameters on a course of the engine's working process, its efficiency and performance as well as ones of the whole propulsion unit - the reciprocating engine generator sets Shu et al., 2013]. A registration of the engine's energy parameters has been complemented with measurements of the chosen exhaust gas components' emission as well as the excess-air-ratio. The measuring data gathered by this way will be used for statistical investigations establishing the significance of an influence of the chemical composition as well as the applied fuel's net calorific value on the selected energy and emission Diesel engine's parameters. In the further perspective there is also planned an application of the statistical tests in order to determine the ranking list of the examined marine fuels. A schematic diagram of the built research test bed, along with places in which the observed control parameters are measured is shown in Fig. 2. Whereas the measuring ranges, exactitudes and sampling periods of the recorded parameters' values are summarized in Table 1.

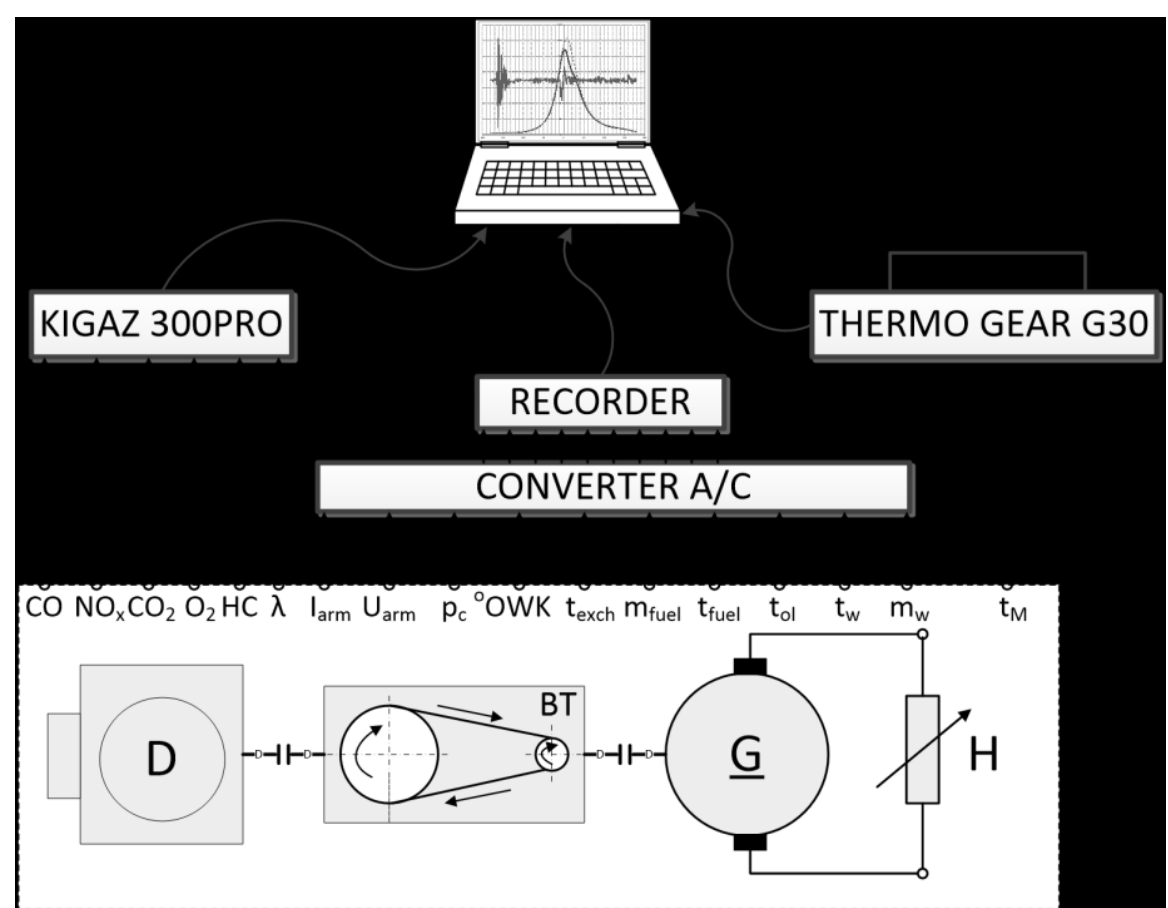

Fig. 2. Schematic diagram of the research test bed with measurement signal conditioning and recording system: $D$ - single-cylinder Farymann Diesel D10 engine;

$\mathrm{BT}$ - belt transmission (multiplier: transmission ratio $\mathrm{i}=\mathbf{0 . 4 2 6}$ ); $\underline{G}$ - direct current generator; $\mathbf{H}$ - heating system 
Table 1

Control parameters of the laboratory propulsion system with Diesel engine

\begin{tabular}{|c|c|c|c|c|}
\hline No & Parameter & $\begin{array}{l}\text { Measurem } \\
\text { ent range }\end{array}$ & $\begin{array}{c}\text { Measurement } \\
\text { Accuracy FS } \\
\text { (\% of Full Scale } \\
\text { Accuracy) }\end{array}$ & $\begin{array}{l}\text { Sampling } \\
\text { period }\end{array}$ \\
\hline 1. & $\begin{array}{l}\text { Rotational speed of the engine's crank- } \\
\text { shaft (angular position - in degrees) }-n\end{array}$ & $\begin{array}{l}0-3000 \\
\mathrm{rev} / \mathrm{min}\end{array}$ & $\pm 0.1 \%$ & $0.5 \mathrm{~ms}$ \\
\hline 2. & Indicated pressure $-p_{i}$ & $0-23.0 \mathrm{MPa}$ & $\pm 3 \%$ & $15 \mu \mathrm{s}$ \\
\hline 3. & Fuel consumption $-m_{\text {fuel }}$ & $0-20 \mathrm{~kg}$ & $\pm 0.2 \%$ & $12.5 \mathrm{~ms}$ \\
\hline 4. & Fuel temperature $-t_{\text {fuel }}$ & $\begin{array}{l}\text { from }-55 \text { to } \\
+125^{\circ} \mathrm{C}\end{array}$ & $\pm 2 \%$ & $93.75 \mathrm{~ms}$ \\
\hline 5. & $\begin{array}{l}\text { Cooling water consumption } \\
\text { (by evaporation) }-m_{w}\end{array}$ & $0-20 \mathrm{~kg}$ & $\pm 0.2 \%$ & $12.5 \mathrm{~ms}$ \\
\hline 6. & Cooling water temperature $-t_{w}$ & $\begin{array}{l}\text { from }-55 \\
\text { to }+125^{\circ} \mathrm{C}\end{array}$ & $\pm 2 \%$ & $93.75 \mathrm{~ms}$ \\
\hline 7. & Lube oil temperature $-t_{o l}$ & $\begin{array}{l}\text { from }-55 \\
\text { to }+125^{\circ} \mathrm{C}\end{array}$ & $\pm 2 \%$ & $93.75 \mathrm{~ms}$ \\
\hline 8. & Exhaust temperature $-t_{\text {exhaust }}$ & $0-350^{\circ} \mathrm{C}$ & $\pm 1 \%$ & $0.1 \mathrm{~ms}$ \\
\hline 9. & Generator's load current (armature) - larm & $0-15 \mathrm{~A}$ & $\pm 1.5 \%$ & $0.1 \mathrm{~ms}$ \\
\hline 10. & Voltage in the armature's terminals $-U_{\text {arm }}$ & $0-250 \mathrm{~V}$ & $\pm 1.5 \%$ & $0.1 \mathrm{~ms}$ \\
\hline 11. & CO content of the exhaust gases & $0-8000 \mathrm{ppm}$ & $\pm 0.1 \%$ & $2 \mathrm{~s}$ \\
\hline 12. & $\mathrm{NO}_{\mathrm{x}}$ content of the exhaust gases & $0-5155 \mathrm{ppm}$ & $\pm 0.1 \%$ & $2 \mathrm{~s}$ \\
\hline 13. & $\mathrm{CO}_{2}$ content of the exhaust gases & $0-99 \%$ & $0.2 \%$ & $2 \mathrm{~s}$ \\
\hline 14. & $\mathrm{O}_{2}$ content of the exhaust gases & $0-21 \%$ & $0.2 \%$ & $2 s$ \\
\hline 15. & $\mathrm{HC}$ content of the exhaust gases & 0-2000 ppm & $\pm 0.1 \%$ & $2 \mathrm{~s}$ \\
\hline 16. & Excess-air-ratio $-\lambda$ & $1-9.99$ & $1 \%$ & $2 s$ \\
\hline 17. & $\begin{array}{l}\text { External surface temperature of the engine } \\
\text { (thermogram) }-t_{M}\end{array}$ & $\begin{array}{l}\text { from }-20 \\
\text { to }+350^{\circ} \mathrm{C}\end{array}$ & $2 \%$ & $1 \mathrm{~s}$ \\
\hline
\end{tabular}

\section{PROGRAMME OF THE ENGINE TESTS}

"How to elaborate the investigation's programme of the engine's working process both in steady and unsteady states?" represents a key metrology question during the testing marine fuels in real operation conditions of a self-ignition engine. In case of the marine diesel engine such an investigation programme should take into account the suitable number of crankshaft rotational speed's as well as load torque's combinations which are determined with a kind of the generated mechanical energy receiver. In the engine's steady states there should be considered three possible relations between the rotational speed and the load torque: propeller, regulatory, speed (Woodyard, 2009). Investigations of the marine fuels will be carried out on a test bed of the Farymann

Diesel D10 type, which drives a separately excited direct current generator of PKM0a44a/143 type through the belt transmission (multiplier of the kinematic transmission ratio equal 0.426 ). Nominal values of the engine's basic parameters amount to: $P_{\text {nom }}=5.9 \mathrm{~kW}, M_{\text {nom }}=38 \mathrm{~N} \cdot \mathrm{m}$, $\mathrm{n}_{\mathrm{nom}}=1500 \mathrm{~min}^{-1}$. Due to limited possibilities of the control and supervision system there was established, that measurements of the observed control parameters characterizing the engine's energy state as well as the emission of exhaust gases will be carried out in 11 different steady load states, laying on the regulatory profile ( 5 states) and propeller profile (6 states). A range of the engine's load with a torque and limited with nominal propeller and regulatory profile was marked out on the basis of basic parameters' nominal values as well as representative values of the engine crank shaft's rotational speed. Finally, taking into account the engine's age (over 50 years) and the waste of a structure of constructional materials, a decision was made to limit a load considerably, which illustrates regulatory c) and propeller d) profiles introduced in Fig. 3. 


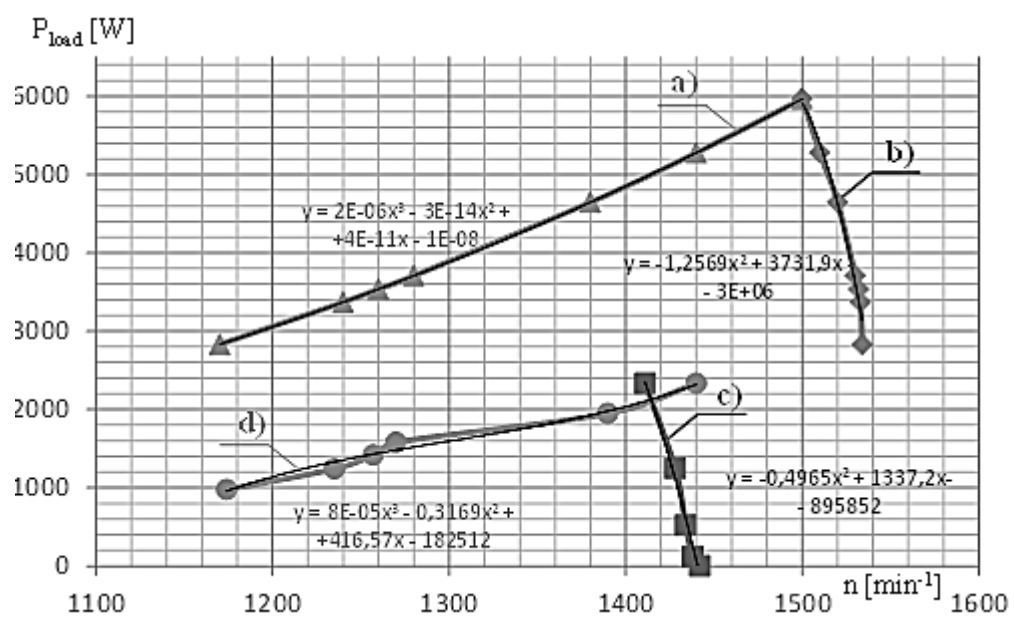

Fig. 3. Profiles of the power alterations of the research engine in term of the crankshaft rotational speed:

a) power-speed propeller profile assigned according to the nominal pair $\left(n, P_{\text {load }}\right)$ nom; b) nominal regulatory profile variable fuel dose at the stabilized by the governor crankshaft rotational speed; c) regulatory profile - according to the test programme being worked out; d) power-speed propeller profile - according to the test programme being worked out

\section{An issue of the engine's thermal equilibrium}

Investigations of a Diesel engine's energy and emission profiles while it is fed with different kind of marine fuels might be realized only after achieving a settled thermal state of its constructional structure. It means, that the cooling system "keeps up" with receiving heat streams from elements of the piston-cylinder group, which directly take over the heat released from the fuel's burning process in the combustion chamber. The larger is a difference between the working medium's temperature and the temperature of walls limiting the combustion chamber the larger energy stream freed in the result of fuel burning is lost on warming the engine's constructional elements. Hence, before starting measurements of the working process's parameters, the engine should be warmed up, bringing it to the state of thermal equilibrium, in which the values of constructional clearances as well as lube oil viscosity will be nominal. In this connection, the long-lasting engine's running at low rotational speed without any load should be avoided, because in such conditions disturbances of spraying and burning process follow $\mathrm{o}-$ in this case: not sufficient and incomplete. They favour creating the carbon deposits in the engine's working spaces as well as in channels of exhaust gases and (alternatively) in a turbocompressor. They also favour growing an emission of toxic chemical compounds in exhaust gases (especially the carbon monoxide). There should be simultaneously considered the fact, that warming-up the lubricative oil goes much more slowly than the one of metal parts being washed with it. Therefore, a state of the engine's thermal equilibrium is defined not with regard to the averaged temperature of its constructional structure's elements or the cooling water's temperature, but with regard to the lubricative oil's temperature.

A comprehensive overview of the available scientific literature confirms that within the period of the engine's warming-up values of the basic parameters values might change in range of a dozen percentage depending on the programme of the engine's load controlling (a dose of the feeding fuel) (Woodyard, 2009). Hence, it was decided that the registration of control parameters begins at the last, 15 minute of the engine's running period at the steady load.

\section{ENGINE ENERGY-BALANCE - STREAM GRAPH}

Energy processes worked out within the marine engine's functional arrangements can be considered in steady and unsteady working conditions of the ship motive or electromagnetic system (Woodyard, 2009; Landeka and Radica, 2016). Limiting the analysis only to the propulsion engine, it is taken an assumption, that conditions of the steady work occur when the derivatives of the state parameters (variables) of the analyzed energy processes in terms of time are equal to zero or they have the settled periodical character (a period is equal to the 
duration of one operation cycle or its multiplicity resulting from the particular cylinders' number). In such a case the processes of energy and mass accumulation or dissipation within the engine's functional arrangements do not occur. Moreover, averaged values of all the input and output energy streams remains idem. In such a case values of the basic parameters, e.g. generated torque, crankshaft rotational speed or summarized heat stream flowing through the engine which are averaged over time corresponding to the realization of the next several operation cycles stay invariable.

An appropriately elaborated stream graph called the Sankey's graph represents a very useful method for a graphic analysis of the energy transformation and transmission processes in engines and working machines, primarily designated to their efficiency estimation (Jamrozik et al., 2017). A stream graph of the energy flow made for the cylindrical section of the Farymann Diesel D10 engine is presented in Fig 4. In such a case the energy balance equation is formulated for the thermodynamic unit which is limited with a balance boundary representing an internal surface of the cylinder. The graph is designed to a simplified analysis of thermal losses of the engine's working process in steady states as well as to make assessment of the engine's thermal efficiency. The widths of individual stream strands presented in the figure above correspond to the streams' values of different energy forms (enthalpy, heat as well as power).

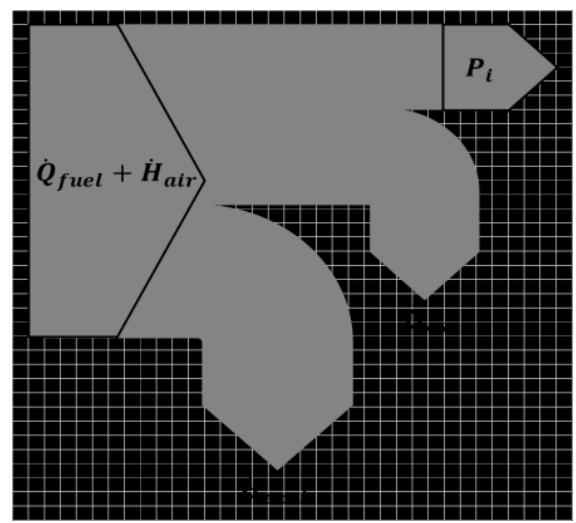

Fig. 4. A Simplified stream graph of the energy flow within the Farymann Diesel D10 engine's cylinder section:

$\dot{Q}_{f u e l}-$ a heat flux entered into the engine along with the power fuel;

$\dot{H}_{a i r}$ - an enthalpy flux of the air feeding the cylinder section;

$\dot{Q}_{c s}-$ a heat flux transmitted with a thermodynamic medium being in the cylinder section to its walls; $\dot{H}_{\text {exch }}$ - an enthalpy flux of the exhaust led out from the cylinder to the atmosphere;

$P_{i}-$ a power generated by the engine in the result of indicating work's realization

A thermal efficiency stands for the basic parameter characterizing the effectiveness of the engine's work in terms of the heat-flow in steady states, i.e. when the averaged values of its effective torque, rotational speed and thermal state are invariable in time. For such the working conditions of work the averaged values of energy streams: input and output for the cylinder section also stay unchanged. In the largest simplifying, the thermal efficiency $\eta_{c}$, stands for a relation of the indicated power to the stream of energy brought into the engine along with a feeding fuel and air, during one operation cycle. Due to the specific construction of the Farymann Diesel D10 engine, in which the cylinder section's cooling is worked out by the water vaporization and the lubricating system is a splash type, the balance boundary was displaced on the surface of the whole engine's external planes.

In such a situation, provided the steady heat flow conditions, a simplifying assumption can be accept, that a sum of all the heat streams, passed from the working medium and circulating mediums (lubricative oil and cooling water) to the walls limited cylinder's internal spaces as well as internal spaces of passages and containers of the lube oil and cooling water, which are built-up into the engine's block, is equal to the heat stream raised to the surroundings from the block's external surfaces by means of radiation. For this reason, a stream graph of the energy 
flow within the examined engine should be respectively modified taking into account its specific construction. There should be considered, except the heat stream radiated to the surroundings from the engine's block $\dot{Q}_{\text {sur }}$ as well as the heat stream taken by the water in a cooling medium's container $\dot{Q}_{W}$, also: the power of mechanical losses in the whole propulsion unit $P_{m}$, the engine's effective power $P_{e}$ which is equal to the generator's propulsion power $P_{G P}$ as well as the stream of the rest heat $\dot{Q}_{r}$ representing the energy losses not considered in the energy balance (e.g. the stream of acoustic energy from mechanical and heat-flow units of the whole propulsion system: the engine-multiplier-generator). On the basis of the above discussion concerning theoretical bases of energy processes worked out within the considered research engine, a block algorithm of calculations processing the results of the conducted measurements into the suitable stream strands of different transmitted and transformed energy forms has been elaborated - Fig. 5. (Woodyard, 2009).

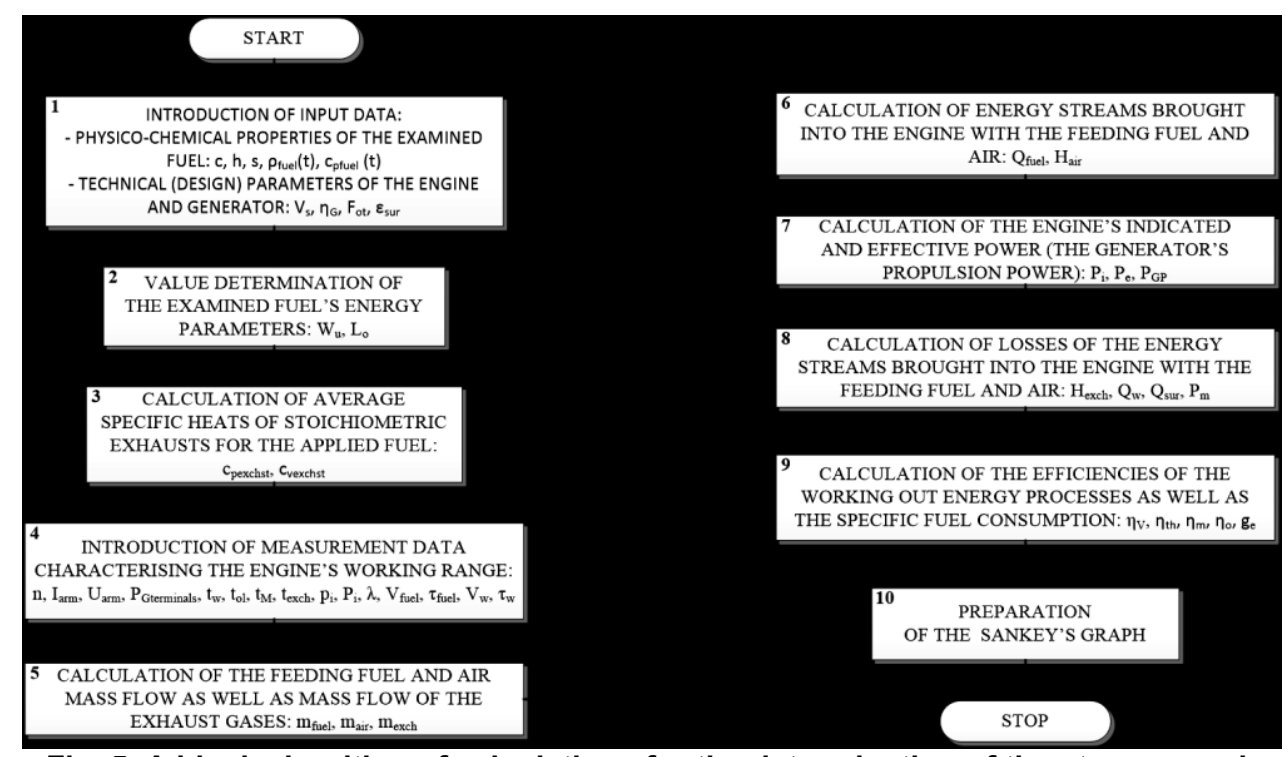

Fig. 5. A block algorithm of calculations for the determination of the stream graph of the energy flow within the propulsion unit with the Farymann Diesel D10 engine powering the direct current generator through the multiplier

The Sankey's graph presented on Figure 6 had been elaborated for one steady state of the unit's load, appointed by symbol $6 s$ on the power-speed propeller profile "d" presented in Fig. 3. The graph represents the ground and diagnostic pattern (defined in specialized literature as a "finger print") for the further comparative analyses of the energy state alterations of the examined engine (and the whole driving unit) fed with different marine fuels.

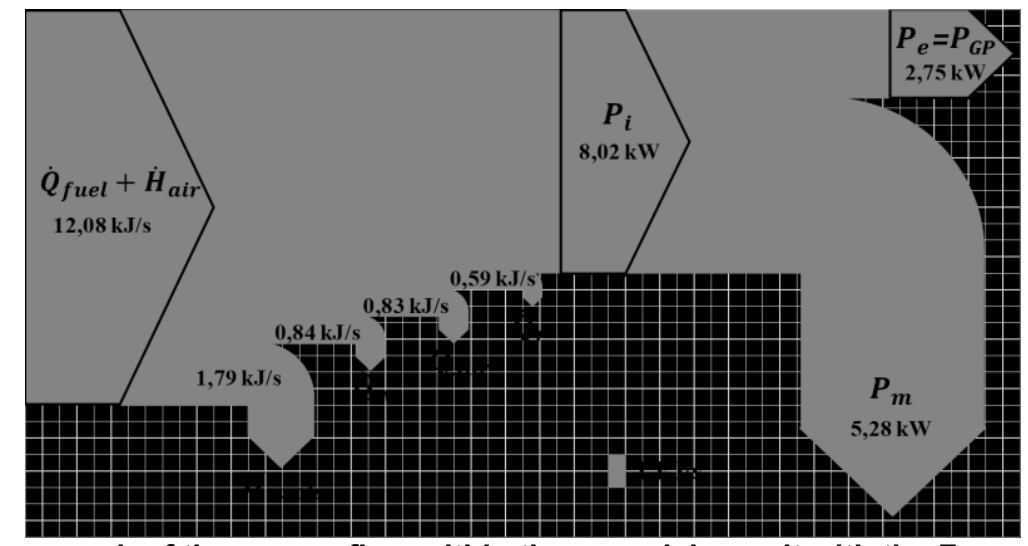

Rys. 6. A stream graph of the energy flow within the propulsion unit with the Farymann Diesel D10 engine powering the direct current generator through the belt drive (multiplier) - elaborated on the basis of control parameters measured at the steady load state $6 s$ of the power-speed propeller profile 


\section{FINAL REMARKS}

In order to carry out a comparative analysis of an impact of the physico-chemical parameters of different marine fuels on the energy state of the examined engine, in a sense of its performance, efficiency and emissivity of the harmful (toxic) substances in exhaust gases, there should be conducted statistical investigations, according to the mathematical model presently elaborated by the author. They will permit to establish the stochastic relations between input and output quantities of the model, on the basis of significance tests of chemical composition's influence as well as the influence of the applied fuel net calorific value on the chosen Diesel engine's energy and emission parameters. Moreover, adequacy tests of the elaborated regress equation by means of Fisher-Snedecor statistics will be worked out. This should consequently lead to the determination of "energy quality ranking" of the examined marine fuels produced by the LOTOS S.A. company (http://www.lotosasfalt.pl/en/1795/products) which is interested in ordering such investigations.

\section{REFERENCES}

Abedin M.J., Masjuki H.H., Kalam M.A.,Sanjid A., Ashrafur Rahman S.M., Masum B.M. (2013). Energy Balance of Internal Combustion Engines Using Alternative Fuels. Renewable and Sustainable Energy Reviews, No 26, p 20-33.

Hürdoğan E. (2016). Thermodynamic Analysis of A Diesel Engine Fueled With Diesel and Peanut Biodiesel. Environmental Progress \& Sustainable Energy, Vol. 35, Issue 3, p. 891897.

Jamrozik A, Tutak W., Pyrc M. and Sobiepański M. (2017) Experimental Investigations on Combustion, Performance, and Emission Characteristics of Stationary $\mathrm{Cl}$ Engine Fueled With Diesel-Methanol and Biodiesel-Methanol Blends. Environmental Progress \& Sustainable Energy, Vol. 36 Issue 4, p.1151-1163.

Korczewski Z., Rudnicki J., Zadrag R. (2017a) Laboratory Station For Research of The Innovative Dry Method of Exhaust Gas Desulfurization for An Engine Powered With Residual Fuel. Combustion Engines. No 168(1), p. 32-37.

Korczewski Z., Marszałkowski K., Rudnicki J. (2017b). The Concept of Research on Ecological, Energy and Reliability Effects of Modified Marine Fuel Oils Application to Supply Compression-Ignition Engines in Real Conditions. Combustion Engines, No 171 (4), p.56-61.

Landeka, P., Radica G. (2016). Efficiency Increase in Ships Primal Energy System Using A Multistage Compression With Intercooling. Thermal Science, Vol. 20, No. 2, p. 13991406.

Shu, G.; Liang, Y.; Wei, H.; Tian, H.; Zhao, J.; Liu, L. (2013). A review of Waste Heat Recovery on Two-Stroke IC Engine Abroad Ships. Renewable and Sustainable Energy Reviews, No 19, p. $385-401$.

Woodyard D. (2009). Pounder's Marine Diesel Engines and Gas turbine. (9th Edition). Elsevier Ltd.

Zeman F., Castaldi M. (2008). An Investigation of Synthetic Fuel Production Via Chemical Looping. Environmental Science \& Technology, No 42 (8), p. 2723-2727.

Marine Fuels Offer - Grupa LOTOS S.A.(2018). Available at http://www.lotosasfalt.pl/en/1795/products [Accessed 2 Apr. 2018] 